\title{
Pflegeberufereformgesetz - zentrales Thema im LV Sachsen-Anhalt
}

Der Landesverband Sachsen-Anhalt trifft sich regelmäßig zu Sitzungen der Schulleitungen, so auch zuletzt am 25. September in der Lutherstadt Wittenberg. Zentrales Thema war und ist das Pflegeberufereformgesetz vom 17.7.2017. Es ist zu erwarten, dass bis zur Einführung der Pflegeberufereform strukturelle und inhaltliche Aufbauarbeit auf Landes- und Schulebene durchzuführen ist. Unklarheiten bestehen hier besonders in der Frage der Zuordnung der Pflegeschulen zum Wissenschaftsministerium (bisher Altenpflegeausbildung) oder dem Ministerium für Arbeit und Soziales (bisher Krankenpflegeausbildung). Der BLGS-Landesverband sieht sich hier in der Position, die Situation der Pflegeschulen im Land in der Zusammenarbeit mit dem Ministerium für Arbeit und Soziales zu stärken. So wurden die Mindestanforderungen an die Pflegeschulen im Land erneut überarbeitet und definiert.

Besonders freut sich der Landesverband über neue Mitgliedsschulen aus der Altenpflege sowie über eine Teilnahme von Mitgliedern aus Sachsen. Die Termine für die nächsten Treffen des Landesverbands sind wie folgt geplant: 20.3.2018 in Magdeburg, 5.6.2018 in Elbingerode, 16.10.2018 in Magdeburg, 11.12.2018 in Halle/Saale.

Christina Heinze

Landesvorsitzende Sachsen-Anhalt

\section{Furiose Auftaktveranstaltung der AG Praxis im LV Brandenburg}

Der BLGS-Landesverband Brandenburg lud am 26.9.2017 zum 1. Brandenburger Praxisanleitersymposium ins Carl-Thiem-Klinikum Cottbus ein. Mehr als 70 Teilnehmer, Praxisanleiter aus verschiedenen Gesundheitsfachberufen und Lehrende aus den Brandenburger Pflegeschulen, folgten der Einladung. Christine Vogler, stellvertretende Bundesvorsitzende des BLGS, stellte die neuen Herausforderungen an die praktische Ausbildung vor, die mit dem neuen Pflegeberufereformgesetz auf die Lehrenden zukommen werden. Folgende Fragen standen im Zentrum der anschließenden Diskussion: Wie kann der Lernort Praxis zukunftsorientiert gestaltet werden? Welche innovativen Ideen der Praxisanleitung gibt es schon? Was sind die drängendsten Probleme bei der praktischen Ausbildung unserer Schüler? Wie können die Anforderungen aus dem Pflegealltag mit den Anforderungen der Ausbildung koordiniert und bewältigt werden?

\section{Didaktische Konzepte zur Praxisanleitung}

Klaus Strimmer vom BLGS-Landesverband Nordrhein-Westfalen stellte mit der Praxisanleitervisite ein Lehr-Lern-Arrangement vor, das an der Fachschule für Gesundheits- und Krankenpflege am Stauferklinikum Schwäbisch Gmünd erprobt und erfolgreich implementiert wurde. Doris Freyberg und Thomas Große, Mit-

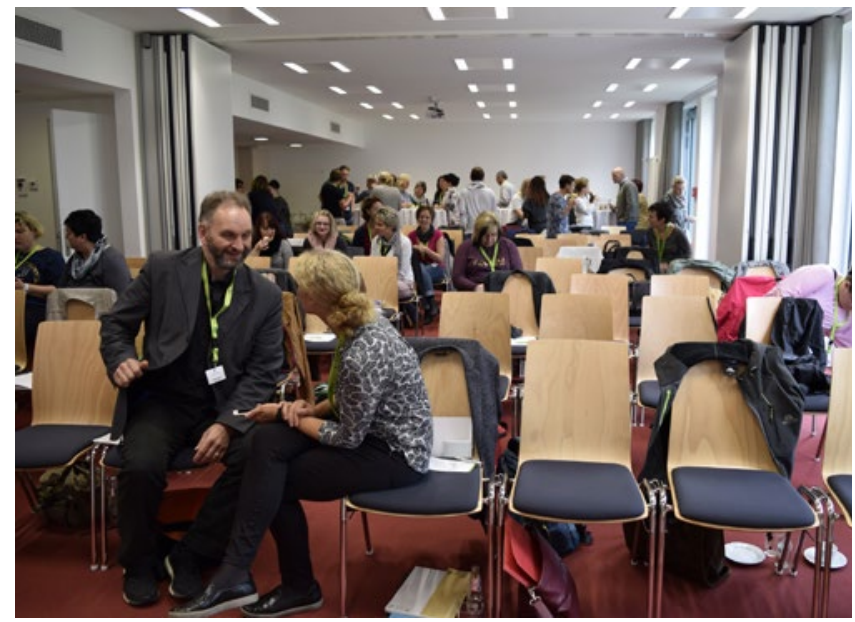

Auch in den Pausen wurde diskutiert glieder der Fachgruppe Praxis des BLGS-Landesverbands Berlin, boten interessante Einblicke in das didaktische Format der Praxistage an der Charité. Doris Schützka, Praxisanleiterin vom BLGSLandesverband Brandenburg, informierte über das Konzept der Praxisanleitung am Carl-Thiem-Klinikum Cottbus.

Mit dem Symposium setzte die AG Praxis einen gelungenen öffentlichen Auftakt ihrer Arbeit im LV Brandenburg, in dessen Rahmen die Teilnehmer viele interessante Anregungen mitnehmen und sich fachlich austauschen konnten. Mit der AG Praxis möchte der Landesverband ein Forum schaffen, in dem sich zukünftig die Praxisanleiter im Land Brandenburg sowohl untereinander als auch mit den Lehrenden in den Pflegeschulen vernetzen können. Mit dem Pflegeberufereformgesetz stehen alle Lehrverantwortlichen vor großen Herausforderungen, die es aktiv und innovativ anzupacken gilt. Der Landesverband Brandenburg wird sich daher auch weiterhin mit den praktischen Erfahrungen aus der AG Praxis und konkreten Vorschlägen bei der Umsetzung auf Landesebene engagieren.

Katrin Pischon Landesvorsitzende Brandenburg

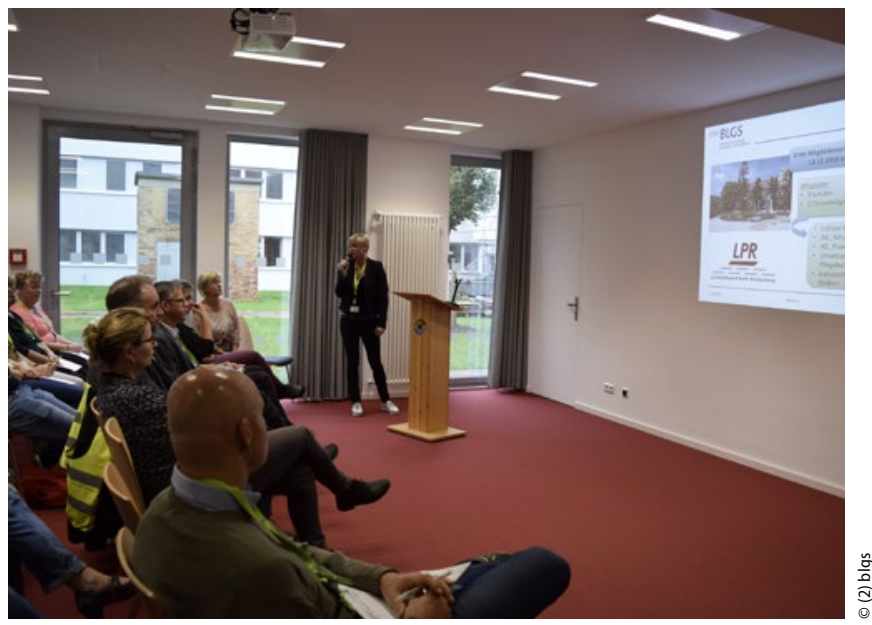

Die stellv. Landesvorsitzende Katrin Gutschlag stellt die Arbeit des BLGS vor 